\title{
Financing railway infrastructure: how to invest and what maintenance policy?
}

\author{
M. Vidaud ${ }^{1}$, O. Bernard ${ }^{1}$, S. Crouïgneau ${ }^{1}$, Y. Putallaz ${ }^{2}$, \\ J. Lévêque ${ }^{3} \&$ R. Jacquier ${ }^{4}$ \\ ${ }^{I}$ OXAND, France \\ ${ }^{2} I M D M$, Switzerland \\ ${ }^{3} R F F$, Laboratoire d'Economie des Transports, France \\ ${ }^{4} R F F$, France
}

\begin{abstract}
This article presents three different ways of predicting investment needs to maintain and renew railway infrastructure. The 'Level 1' approach is based on financial ratios and does not take into account the infrastructure's history or maintenance strategies. This simplified approach can be used provided that the components age pyramid is uniform. The 'Level 2' approach considers the age pyramid's general impact without integrating budget constraints and possible specific optimization at the component level. This approach is useful and gives interesting rough estimates for macroscopic finance audits; it does not require an exhaustive knowledge of the owner's material assets. The advanced 'Level 3' approach enables planning of detailed investment needs on short, medium and long-term scales. It considers both physical and economic lifetimes for each component of the infrastructure as well as taking into account the budget constraints of the infrastructure's manager. It also allows the possibility of quantifying risks related to performance failure due to underinvestment. Using these different approaches, or cost models, with different European infrastructure managers enabled demonstration that investment needs and thus potential regulated returns (for regulated railway network management), strongly depend on infrastructure history and components age pyramid as well as physical and economic lifetimes. The article shows that management policy decisions and balance between renewal and maintenance, crucial in securing long-term network integrity (or 'substance') and in deciding on short-term investments, can
\end{abstract}


be pre-modelled using advanced methods and tools to assess performance versus cost and risk over time.

Keywords: railways, asset management, investment needs of infrastructure, CAPEX, OPEX, age pyramid.

\section{Introduction}

Since the 91/440/EC directives, a railway reform was launched in the European Union in order to improve the competitiveness of the railway transport market by strengthening supervision.

In the current situation of the financial and economic crisis, raising investment becomes increasingly difficult as governments tend to reduce subsidies and private investment funds set high interest rates.

In this context, it is more tempting than ever to reduce the level of investment. Underinvestment does not have impact in the short term but is very risky in the medium to long term and can lead to safety public concerns. It is today known that several railways networks have suffered of chronic underinvestment in the past decades.

The ability of setting investment and maintenance policies based on performance levels and life cycle cost optimisation is crucial. To succeed, infrastructure managers continuously shift from a rather technical approach towards a more cost and performance oriented approach, to be able to justify and to support decisions more clearly.

Asset management should help determine the investment needs and the level of risk incurred if the amount of investment is too low. At least three actors are involved in making the decision of defining the right investment needs and should then take part in this process:

- the infrastructure owner, who defines the expected level of performance

- the engineers or technical specialists: they translate the owner's requirements into technical specifications and manage the technical life cycle of assets

- the financial manager; he integrates activities linked to infrastructure financing into an accounting framework and manages the accounting life cycle of assets

This article aims to present three different methods to assess the investment needs as asset management support: a simplified financial approach, an agebased approach, and a more advanced approach based on asset management outputs. This last approach strengthens the link between technical and financial considerations.

\section{Level 1: simplified financial approach}

A simplified approach to evaluate an investment policy can be based on the accounting of assets. Theoretical annual investment needs are obtained by dividing the gross value of the asset by their expected technical lifetime (time at which it is economically no more reasonable to maintain the component; at the 
end of this lifetime, the component is renewed), given that every component is renewed at the end of this lifetime. For numerous infrastructures, this can be calculated by homogenous types of asset. These theoretical annual investment amounts can be compared to the real level of depreciation [1].

Let's notice that in the whole paper, depreciation as well as gross value should be considered as taking into account price evolution. The approaches discussed in this paper suppose either there is no inflation, either "depreciation" is economic depreciation based on the current value of investments, rather than accounting depreciation based on historic value of investments

In the context of a uniform age pyramid and a stabilized renewal policy, the net book value should be constant over the years. Thus, annual investment needs should be equal of the annual depreciation: net book value $=0.5^{*}$ (accounting lifetime/expected technical lifetime)* gross value (if both accounting lifetime and expected technical lifetime are equal, then the net book value $=0.5^{*}$ gross value).
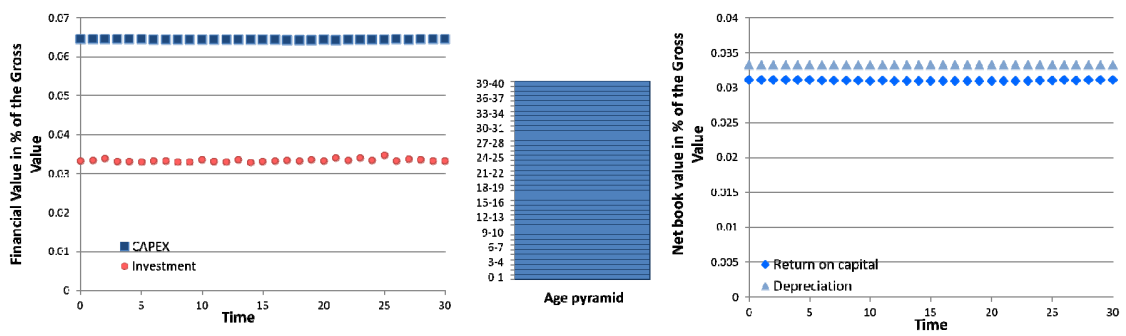

Figure 1: Evolution of investment needs and capital expenditure (CAPEX) for a fictive set of asset in a stabilized renewal context with a uniform age pyramid. Accounting lifetime=expected technical lifetime $=30$ years. Capital expenditure is the sum of depreciation and return on capital (return on capital $=\mathrm{WACC}^{*}$ Net Book Value). WACC $=6.2 \%$ (WACC used by Réseau Ferré de France to calculate return on capital for its minimal services [2]).

The main hypothesis of this method is that the renewal policy has been stabilized for a long time and that the age pyramid of the assets is quasi-uniform. This could be the case of large networks where the investment has been rather stabilized over the years.

If theoretical annual investment needs are different than real investment budgets then one should deepen the analysis:

Theoretical needs are above investment budgets:

- Is that the effect of a slight trunk pyramid?

- Is the network losing its substance?

Theoretical needs are below investment budgets:

- Is that the effect of a slight trunk pyramid?

- Is there any change of technology that requires more investments?

- Are the accounting lifetimes coherent with the expected technical lifetimes? 
This method is simple to use and may give quick answers to some strategic questions about investments policies. Though, as soon as the structure of the age pyramid is heterogeneous, one should consider integrating that heterogeneity in the evaluation.

\section{Level 2: financial approach integrating age pyramid form}

A more precise estimation regarding the "right level of investment" has to take into account the age pyramid. If the asset database is not precisely known, one should make assumptions (based on expert views) on the shape of the pyramid of age.

Using the shape of an infrastructure age pyramid (or a homogenous part of it), it is possible to simulate the life cycle of a component, for example with a Monte Carlo method. The initial age is determined by a stochastic variable that has the shape of the age pyramid of infrastructure.

If at the end of its technical lifetime, the component is automatically renewed, then the depreciation is constant. In this case the investment needs to follow the shape of the age pyramid [1].

For an inverse triangular shaped pyramid (some new components, but mostly old components), the investment needs at year 0 is $2^{*}$ gross value/technical lifetime. Up until the midpoint of the technical lifetime, the investment needs are higher than the depreciation, but once the midpoint is passed the trend gets inversed.
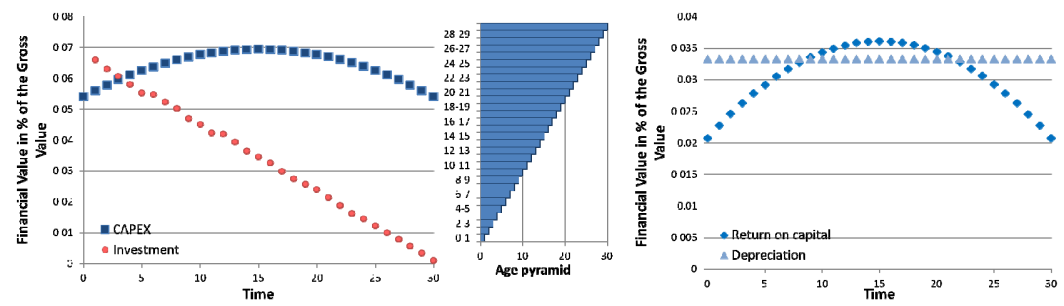

Figure 2: Evolution of investment and capital expenditure for a fictive set of asset, with an inverse triangular shaped pyramid, expected technical lifetime $=$ accounting lifetime $=30$ years. WACC $=6.2 \%$.
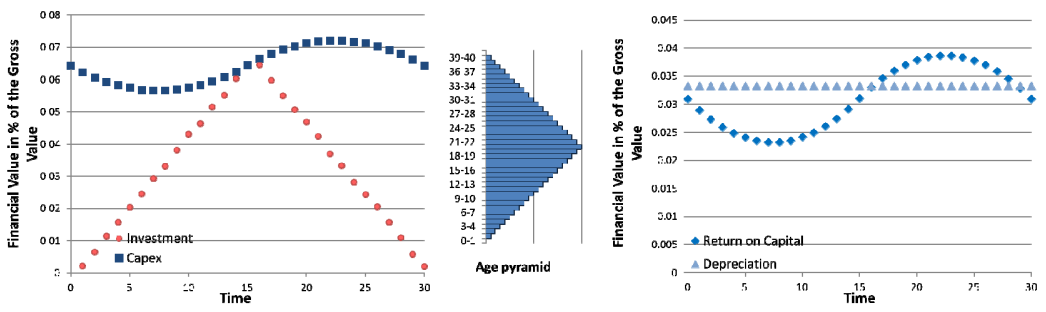

Figure 3: Evolution of investment and capital expenditure for a fictive set of asset with a symmetrical shaped pyramid, expected technical lifetime $=$ accounting lifetime $=30$ years. $\mathrm{WACC}=6.2 \%$. 
For a symmetrically shaped pyramid, the highest investment is needed during the middle of the technical lifetime. At this point the investment is equal to $2^{*}$ gross value/technical lifetime.

Accounting lifetimes are usually lower than technical lifetimes (they are set following accounting principles). Therefore, investment needs are distributed on a larger period and depreciation is not constant anymore.

Let's notice that in some regulated context, depreciated components do not generate investment remuneration anymore.
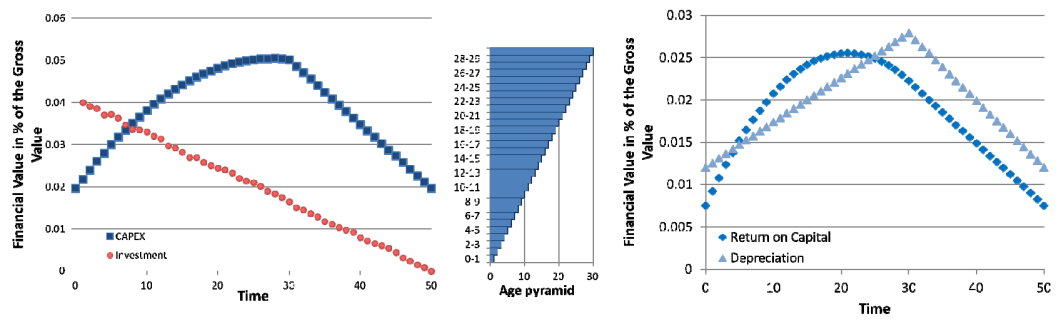

Figure 4: Evolution of investment and capital expenditure for a fictive set of asset, with an inverse triangular shaped pyramid, expected technical lifetime $=50$ years, accounting lifetime $=30$ years. $\mathrm{WACC}=6.2 \%$.
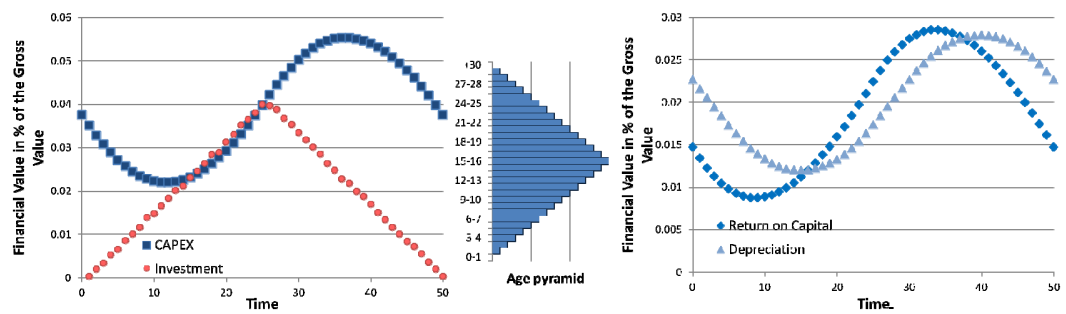

Figure 5: Evolution of investment and capital expenditure for a fictive set of asset for a fictive set of asset with a symmetrical shaped pyramid, expected technical lifetime $=50$ years, accounting lifetime $=30$ years. $\mathrm{WACC}=6.2 \%$.

As long as one has some knowledge about the form of the pyramid of age, this approach may significantly improve the accuracy of the investment policy evaluation. But, the approach still relies on the hypothesis that investment budgets were rather stabilized in the past. Thus, especially on ageing networks, one has to look to more complex approach, based on a more precise knowledge of the assets.

\section{Level 3: advanced approach - financial approach based on asset management outputs}

The added-value of this third approach is the ability of integrating asset budget constraints (in the past and projections) in the investments policy evaluation. 
Moreover, the method ensures a thorough integration of asset management concepts (asset knowledge, data and financial considerations).

The approach relies on a forecasting tool able to predict investment needs under budget constraints, based on an asset database and specific maintenance models. The simulation tool provides a vision of obsolescence effects and of corresponding risk evolution.

This chapter presents two case studies.

In the first case study, the aim is to analyse the combined impact of maintenance strategy and budget constraint on a track section.

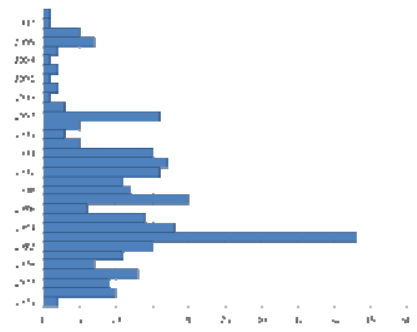

Figure 6: Age pyramid of a track section.

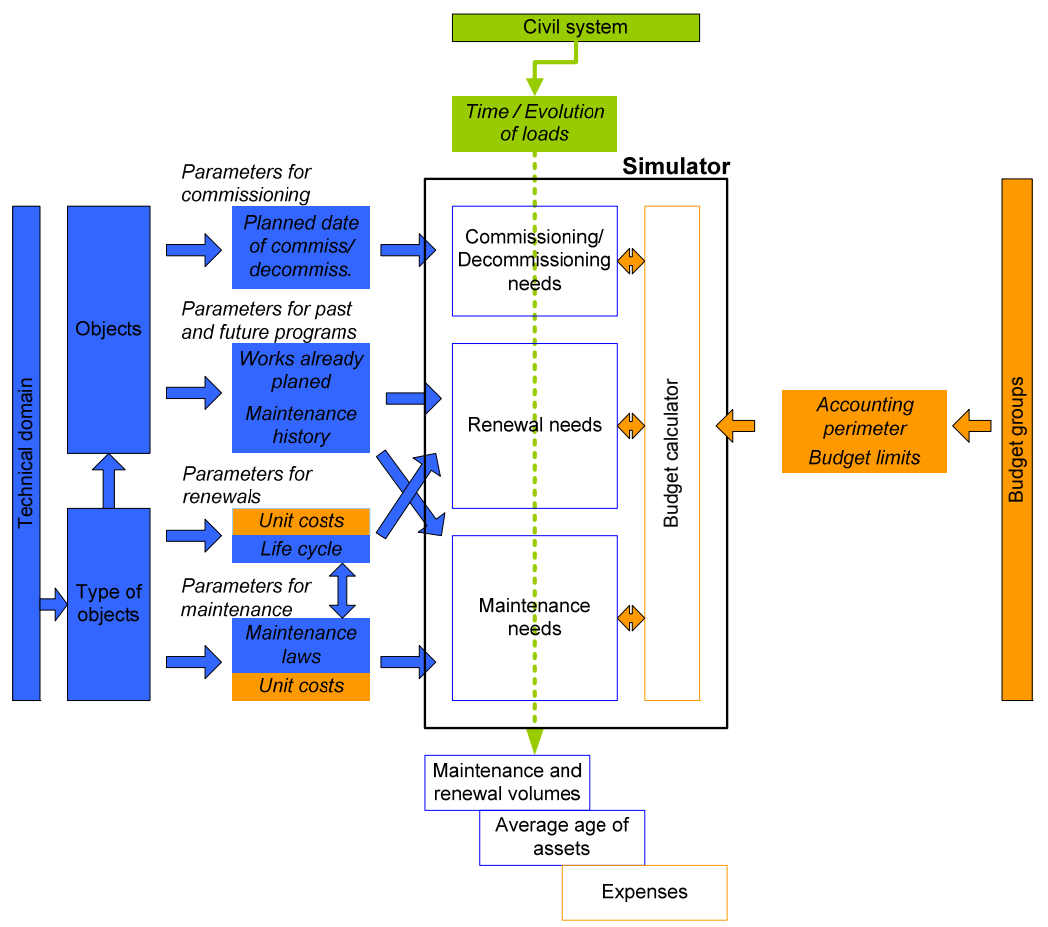

Figure 7: Working diagram of $\mathrm{SIMEO}^{\mathrm{TM}}$-StrateGO tool 'asset module'. 
Investment needs have been determined using an advanced approach with the aid of SIMEO ${ }^{\mathrm{TM}}$-StrateGO tool, developed by Oxand. This method considers the life cycle of each component and their maintenance plan as well as taking into account budget constraints. Budget constraints can lead to yearly equal expenses: if budget is not sufficient, best compromise between investment postponement and investment needs is to be found.
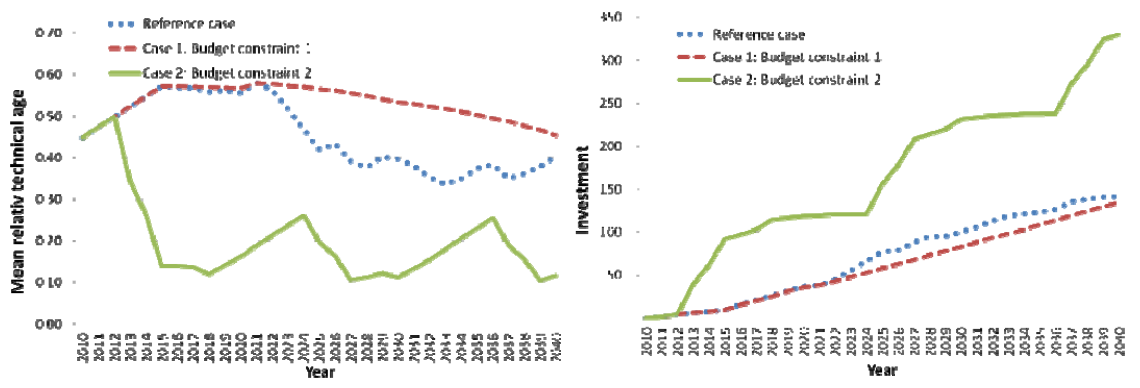

Figure 8: Evolution of the mean relative age and of investment needs on a track section. In case 1, renewal budget is constrained; in case 2, maintenance budget is constrained.

The higher the investment budget, the lower the mean relative age (ratio age/technical lifetime by the unit cost of renewal of component). The type and amount of budget constraints directly affect the mean relative age.

It is easy to compare two "extreme" investment strategies in track. Case 1: unlimited budget for maintenance and restriction on renewals and Case 2: the inverse of Case 1.

The Reference Case represents a real situation in order to check the relevance of the modelling of maintenance law.

Case 1 stabilises the mean relative age to 0.58 and makes it lower from 2020 on; after 2036 the mean relative age is lower than 0.5 . The investment amount is the same as the Reference Case until 2020 and is lower afterwards.

In case 2, the track section gets almost "new", but investments are high compared to the Reference Case.

The aim of the second case study is to assess risk on a catenary network according to different scenarios of budget constraints: no investment (scenario 0 ), budget constraint $\mathrm{X} /$ year (scenario 1 ), investment=depreciation (scenario 2), budget constraint $\mathrm{Y} /$ year with $\mathrm{X}<\mathrm{Y}$ (scenario 3), unlimited budget (scenario 5). This analysis also used the SIMEO- StrateGO tool.

Scenario 1 leads to the obsolescence of the network, ratio of risky catenaries increase from $10 \%$ to $25 \%$. Scenario 2 (investment=depreciation) stabilizes the mean relative age to about 0.6 but does not enable catching up the investment delay. Scenario 3 reaches a mean relative age 0.5 after 30 years and maintains the risky catenary length under $10 \%$ of the total catenaries length. 

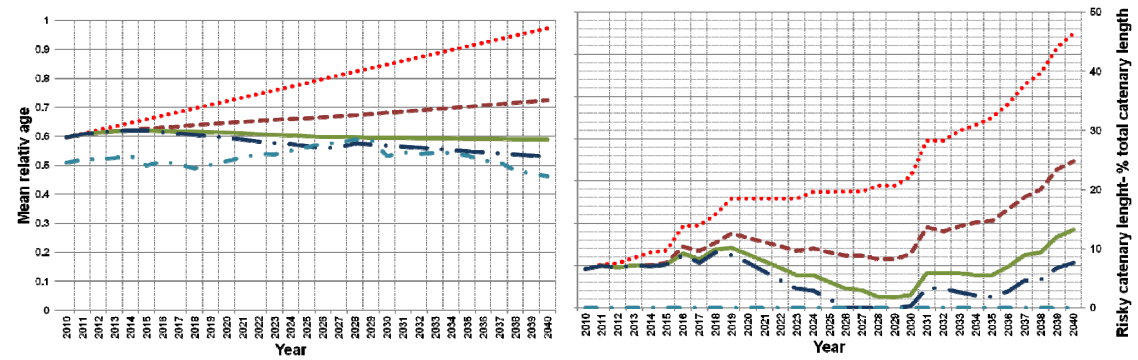

...... Scenario 0 : No investment _ _ Scenario 1: budget constraint 1

Scenario 2: Investment $=$ Depreciation

- Scenario 3: budget constraint $3-$ Scenario 5: No budget constraint

Figure 9: Evolution of the mean relative age and "risky length" of the catenary according to different investment budget scenarios.

Note: a risky catenary length has been defined when its age becomes higher than its expected technical lifetime in the coming 30 years.

As shown on and, stopping the catenaries investment will be a disaster (Scenario 0), and Scenario 3 will lead to an increase of the Net Book Value of ca. $20 \%$ in a term of 30 years.

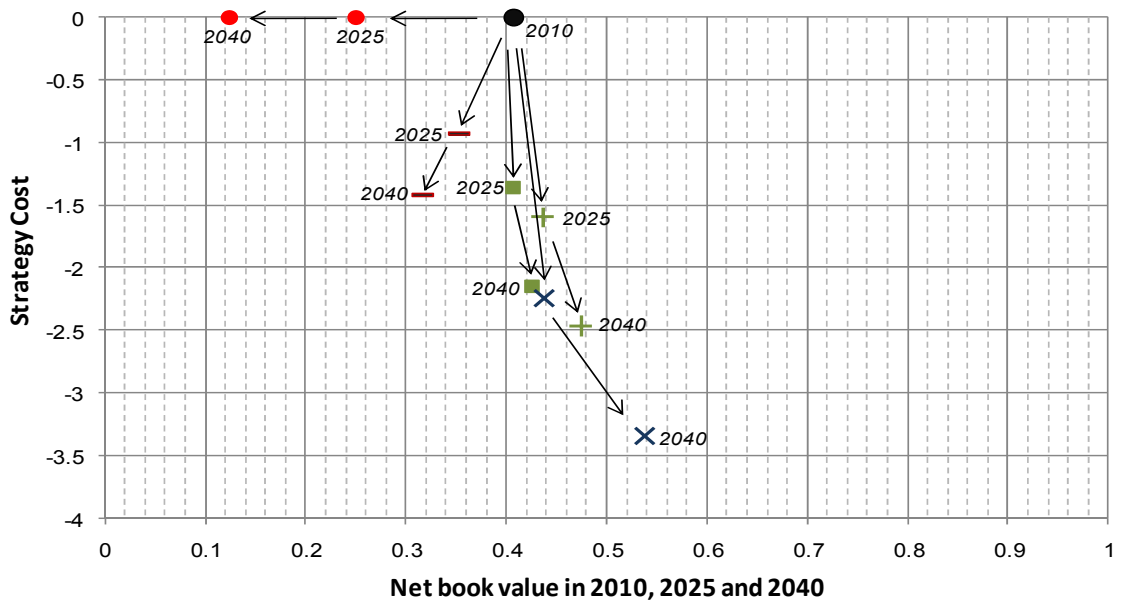

Scenario 0: No investment $\quad$ - Scenario 1: budget constraint 1 Scenario 2: Investment = Depreciation

+ Scenario 3: budget constraint $3 \times$ Scenario 5: No budget constraint

Figure 10: Evolution of the net book value testing different scenarios.

Testing the effect of different strategies of investment, maintenance and expected parameters (lifetimes) on performance is the key strength of this 
advanced method [1]. It also allows the analysis of the right balance between renewals and maintenance activities.

Performance and risk analysis use the mean relative age of the components: the level of risk posed by an obsolete component for an infrastructure depends on its type; the consideration of an intervention can be then determined. The cost of risk can also be calculated.

Moreover, it also makes possible to calculate the accurate value of remuneration of CAPEX and to analyse investment strategy influence on operating cost.

\section{Findings}

Approach 1 can provide an order of magnitude for the investment needs of an infrastructure fleet over a given period but is accurate only if renewals have been stabilized and pyramid age is roughly uniform.

Approach 2 can provide the investment needs during a period taking into account the shape of age pyramid. The output can be used for an audit on financing, it provides an overview on investments and accounting practices. Nevertheless, it does not take into account budget constraints and their impact on the performance of the infrastructure.

Approach 3 can be used for detailed investment budget planning, for the short, medium and long term. This approach requires the commissioning date, technical and accounting lifetimes, and renewal costs for each component. Using this approach, it is also possible to implement maintenance strategy and analyse the risk of performance decrease in the case of underinvestment.

Investment needs are used to calculate remuneration of CAPEX. The Level 1 and 2 approaches give an idea of their evolution and allow the comparing of scenario trends but cannot provide a precise amount or operating cost evolution. The advanced approach Level 3 can precisely calculate remuneration on CAPEX and analyse the impacts of the investment strategy on operating cost. When infrastructure managers and public (or private) investors come to negotiations for subsidies: investment strategies are key factors.

\section{References}

[1] Bernard O., Vukicevic A, Oxand Suisse, Service Électrique de Lausanne, Financement des infrastructures publiques; Combien investir et quelle rémunération? (to be released in 2012).

[2] $R F F$, Network statement 2013, appendix 10.

[3] Putallaz Y., Rivier R. - EPFL - Swiss Federal Institute of Technology, Lausanne, Strategic evolution of railways corridors infrastructure: Dual approach for assessing capacity investments and M\&R strategies (2004). 